\title{
Mitogen-activated protein kinase pathway is pivotal for anoikis resistance in metastatic hepatoma cells
}

\author{
LILI CAO $^{1}$, ZHIYONG ZHANG ${ }^{2}$, LIHUI HAN ${ }^{2}$, JUAN DU ${ }^{1}$, \\ XIAOHONG LIANG ${ }^{2}$, YUGANG LIU ${ }^{3}$ and WENSHENG SUN ${ }^{2}$ \\ ${ }^{1}$ Medical Research Center, Shandong Provincial Qianfoshan Hospital, Shandong University, \\ Jinan, Shandong 250014; Departments of ${ }^{2}$ Immunology and ${ }^{3}$ Pathophysiology, School of Medicine, \\ Shandong University, Jinan, Shandong 250012, P.R. China
}

Received September 30, 2013; Accepted January 29, 2014

DOI: $10.3892 / \mathrm{mmr} .2014 .1952$

\begin{abstract}
It is important for metastatic cancer cells to acquire anoikis resistance for survival in the circulatory system. In the present study, metastatic hepatoma cells were demonstrated to acquire anoikis resistance, which renders them more invasive, more resistant to anticancer agents and able to evade the host immune system for long-term survival. One of the most significant characteristics of these anoikis-resistant metastatic hepatoma cells is their proliferation inhibition. However, when microarray results were analyzed to identify the underlying molecular mechanism, the mitogen-activated protein kinase (MAPK) signaling pathway was found to be markedly upregulated, which appeared to conflict with the proliferation inhibition state. To investigate this result and the associated mechanism, protein kinase inhibitors were used to inhibit the phosphatidylinositol 3-kinase (PI-3K)/AKT and MAPK pathways. It was found that anoikis-resistant hepatoma cells may compensate for the inhibition of PI-3K/AKT or MAPK pathways by cross-talk between these two pathways, which increases their survival capacity during metastasis. In concordance with this result, western blot analysis revealed that the phosphorylation level of extracellular signal-related
\end{abstract}

Correspondence to: Professor Lili Cao, Medical Research Center, Shandong Provincial Qianfoshan Hospital, Shandong University, 16766 Jingshi Road, Jinan, Shandong 250014, P.R. China

E-mail: cllly22@163.com

Professor Wensheng Sun, Department of Immunology, School of Medicine, Shandong University, 44 Wenhuaxi Road, Jinan, Shandong 250012, P.R. China

E-mail:wsw@sdu.edu.cn

Abbreviations: PI-3K, phosphatidylinositol 3-kinase; MAPK, mitogen-activated protein kinase; ERK, extracellular signalregulated kinase; CCK8, cell counting kit-8; ECM, extracellular matrix; Poly-HEMA, poly(2-hydrocyethyl methacrylate; FDR, false discovery rate; Re, the enrichment; Path-Net, pathway-network

Key words: mitogen-activated protein kinase pathway, anoikis resistance, metastasis, survival, hepatoma, cancer kinase protein was increased when the PI-3K/AKT pathway was inhibited. Therefore, it was concluded that when metastatic hepatoma cells aggregate in blood vessels, proliferation is inhibited and the MAPK signaling pathway is upregulated, which increases the long-term survival of the cells. Furthermore, a compensatory interplay between the AKT and MAPK signaling pathways was observed in the present study. Using kinase inhibitors for the two pathways in combination may yield a substantial advance in successfully producing a downstream phenotypic response in anoikis-resistant metastatic hepatoma cells.

\section{Introduction}

Hepatocellular carcinoma (HCC) is the fifth most common type of neoplasm worldwide, as well as the third most common cause of cancer-related mortality (1). Patients usually succumb to metastases rather than primary HCC. Thus, understanding the molecular mechanisms of metastasis is of crucial importance for HCC therapy. It is well known that cancer cells have to accomplish a series of sequential steps to establish distant metastases, including detachment from the primary tumor, invasion into and survival in the vasculature, extravasation into the stroma of different organs, and proliferation in the secondary site. Each of these steps can be potentially targeted for treatment, but limited knowledge regarding the molecular mechanisms of the metastatic steps renders the majority of therapeutic strategies largely inefficient (2). Therefore, it is important to investigate the molecular mechanisms of each metastatic step. The majority of metastasis studies have focused on the primary tumor and the organ to which it has metastasized (3), known as the first microenvironment and secondary microenvironment, respectively; the importance of the circulatory system, known as the third microenvironment, in tumor metastasis has only recently been recognized (4). Although certain studies have focused on metastatic mechanisms of cells in the third microenvironment (5-7), and other studies have demonstrated that metastatic tumor cells are able to survive for a long time by forming homotypic multicellular aggregates $(8-10)$, it is largely unknown how these aggregated cells survive in the anchorage-independent third microenvironment. 
Anoikis, a Greek word meaning 'loss of home' or 'homelessness', was first coined by Frisch and Francis in 1994 to describe a type of apoptosis that occurs when adherent cells are deprived of anchorage (11); it was later recognized as a potentially important element in tumor angiogenesis and metastasis (12-14). Resistance to anoikis enables malignant cells to survive in an anchorage-independent manner and increase their survival time, as well as facilitating their eventual reattachment and colonization at secondary sites. Acquisition of anoikis resistance is known to be critical for cancer metastasis. However, the mechanisms leading to aberrant survival vary greatly among cell types (5). Understanding the mechanisms of anoikis resistance may greatly benefit the development of efficacious treatments for cancer. Our previous study reported that hepatoma cells were able to resist anoikis through novel synoikis-like survival (8). Synoikis describes the process in which cancer cells form a multicellular aggregate to maintain survival and proliferation after detachment. We also reported that hepatoma cells acquired greater metastatic potential when they were suspended in extracellular matrix (ECM) and acquired the ability for anoikis resistance (15).

The phosphatidylinositol 3 kinase (PI-3K)/AKT and mitogen-activated protein kinase (MAPK) signaling pathways have been well investigated. They are the most dominant proliferation and survival signaling pathways. These two survival pathways are known to be inhibited by a loss of adhesion $(16,17)$. One of the predominant changes observed in circulating tumor cells is the loss of adherence to neighboring cells and the basal membrane. The AKT pathway has been shown to be important in mediating signals that lead to anchorage-independent survival, and inhibition of the PI-3K/AKT pathway has been shown to lead to anchorage-independent cell death, or anoikis $(18,19)$. AKT and extracellular signal-regulated kinase (ERK) protein kinases are important in signaling pathways that respond to growth factors and other extracellular stimuli. They regulate several cellular functions, including nutrient metabolism, differentiation, cell growth, proliferation, apoptosis and survival. One study has reported that treatment with ERK and PI-3K inhibitors significantly inhibited the invasiveness of hepatocellular carcinoma cells (20).

Whilst a number of studies investigating anchorage-independent survival mechanisms have focused on the PI-3K/AKT and MAPK pathways in different types of malignancy (21-24), there is little information regarding hepatoma cells. Therefore, in the present study, the protective effect of the PI-3K/AKT and MAPK pathways upon anchorage removal in the synoikis-like hepatoma cells was specifically examined. The molecular mechanisms underlying resistance to anoikis in metastatic hepatoma cells were investigated using protein kinase inhibition.

\section{Materials and methods}

Cell culture. BEL7402 human hepatoma cells were routinely cultured and maintained in RPMI 1640 medium supplemented with $10 \%$ fetal calf serum in the presence of $5 \% \mathrm{CO}_{2}$ at $37^{\circ} \mathrm{C}$. To obtain the metastatic cell model, the cells were seeded into plates with a poly-2-hydroxyethyl-methacrylate (HEMA; Sigma-Aldrich, St. Louis, MO, USA) coating, as described previously (15). A trypan blue exclusion assay (Beyotime,
Nantong, China) was used to count the number of dead cells and a cell counting kit-8 (CCK8) assay (Dojindo, Kunamoto, Japan) was used to determine the cell viability as described previously (8). All the experiments described in the current study were performed in triplicate and repeated three times.

Microarray. RNA extraction and cDNA synthesis were performed as described previously (15). Human oligonucleotide probe arrays (CapitalBio Corporation, Beijing, China) were applied to detect the mRNA expression levels of 22,000 transcripts. cDNA samples from attached and detached groups were labeled with $\mathrm{Cy} 3$ and $\mathrm{Cy} 5$ fluorescent dyes (Amersham Pharmacacia Biotech, Piscataway, NJ, USA), respectively. DNA microarray scanning was performed using a LuxScan 10KA Microarray Scanner (CapitalBio Corporation). The images were analyzed using GenePix Pro 4.0 software (Molecular Devices, Sunnyvale, CA, USA) and saved as Excel files.

microRNA array. miRNAs were labeled using the miRCURYTM Array Labeling kit (Exiqon, Vedbaek, Denmark) and the labeled sample was concentrated using the RNeasy Mini kit (Qiagen, Dusseldorf, Germany). miRNA array hybridization was performed using the miRCURY LNA $^{\mathrm{TM}}$ microRNA Array kit (Exiqon, Vedbaek, Denmark). Images were acquired by scanning the slide using the Genepix 4000B (Molecular Devices). The data were analyzed by Genepix Pro 6.0 software (Molecular Devices) and saved as Excel files.

Analysis of the array results. Pathway analysis was used to identify significant pathways of the differentially expressed genes according to the Kyoto Encyclopedia of Genes and Genomes (KEGG; http://www.genome.jp/kegg/), BioCarta (BioCarta LLC, San Diego, CA, USA; http://www.biocarta. com/) and Reactome (http://www.reactome.org/). Fisher's exact test and $\chi^{2}$ test were used to select the significant pathways, and the threshold of significance was defined by $\mathrm{P}$-value and the false discovery rate. The enrichment was calculated according to published studies (25-27). The pathway-network was the interaction net of the significant pathways of the differentially expressed genes built according to the interaction among pathways of the KEGG database to find the interaction among the significant pathways directly and systemically. It was able to summarize the pathway interactions of differentially expressed genes in certain diseases and identify why certain pathways were activated (26). The networks of genes in different signaling pathways (Signal-net) were analyzed using the KEGG database to build a network of genes according to associations among the genes, proteins and compounds in the database (28-32). The association of the microRNA and genes was calculated using their differential expression values, and the MicroRNA-Gene-Network was built according to the interactions of microRNA and genes in the Sanger microRNA database (Wellcome Trust Sanger Institute, Hinxton, UK). The adjacency matrix of the microRNA and genes, $A=[a i, j]$, was constructed as determined by the attribute associations among genes and microRNA; ai,j represents the relation weight of gene i to microRNA j. 

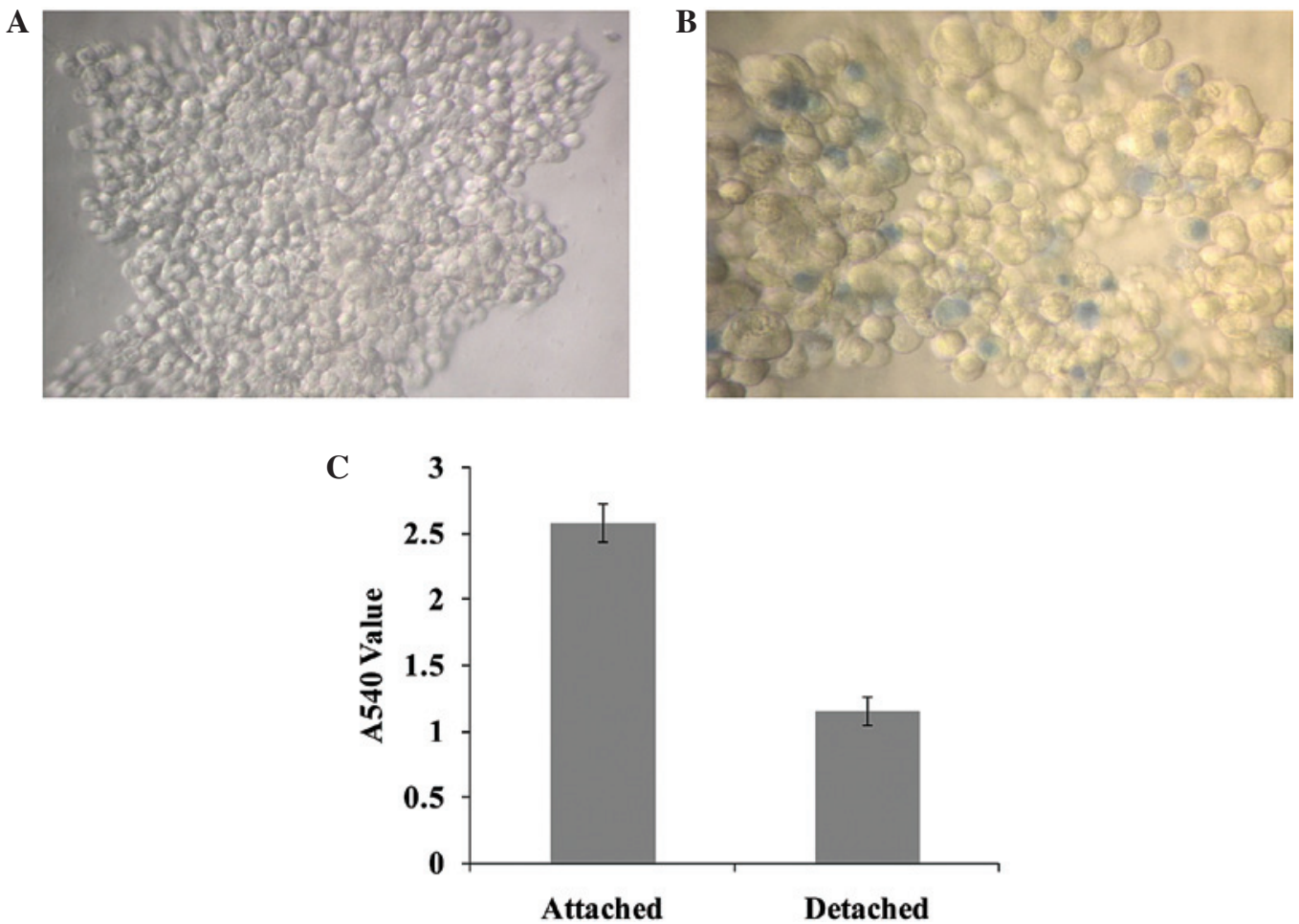

Figure 1. Viability of the detached BEL7402 human hepatoma cells. (A) Morphology of the detached cells. When cells were detached, they gathered into aggregates to resist anoikis and therefore survived. (B) Morphology of live detached cells with no staining; dead cells were stained using trypan blue. Few cells died in the aggregates (magnification x20). (C) Cell viability of attached and detached cell groups detected by cell counting kit-8 assay. Detached cells proliferated markedly slower than the attached group. Each experiment was performed in triplicate and all experiments were repeated three times with similar results. $\mathrm{P}<0.05$, vs. attached cells.

AKT or ERK inhibition. BEL7402 cells were seeded in 96-well or 6-well plates with or without a poly-HEMA coating as detached or attached cells, as described above. The detached and attached cells were treated with the PI-3K/AKT pathway-specific inhibitor Wortmannin (Cell Signaling Technology, Inc., Danvers, MA, USA) or the ERK pathway specific inhibitor PD98059 (Cell Signaling Technology, Inc.). The cells in either adherent or suspension conditions were treated in triplicate with either $1 \mu \mathrm{M}$ wortmannin, $50 \mu \mathrm{M}$ PD98059 or both for $24 \mathrm{~h}$. The cell viability, cell death rate and protein expression level were then determined using the CCK8 kit, trypan blue exclusion assay and western blot analysis, respectively.

Western blot analysis. Cells were washed three times in phosphate buffered saline and incubated in lysis buffer $(10 \mathrm{mmol} / 1$ TrisCl, $8 \mathrm{M}$ urea, $4 \%$ CHAPs, $1 \%$ DTT, $0.3 \mathrm{mg} / \mathrm{ml}$ EDTA and $35 \mu \mathrm{g} / \mathrm{ml} \mathrm{PMSF}$ ) at $4^{\circ} \mathrm{C}$ for 20 minutes. Equal quantities of protein from each lysate were subjected to $10 \%$ SDS-PAGE and transferred to a nitrocellulose membrane. Subsequent to blocking for $1.5 \mathrm{~h}$ in $5 \%$ non-fat dried milk containing $0.1 \%$ Tween 20 , the membrane was incubated at $4^{\circ} \mathrm{C}$ overnight in the presence of phospho-p44/42 MAP Kinase (Thr202/Tyr204) antibody (Cell Signaling Technology, Inc.), p44/42 MAPK antibody (Cell Signaling Technology, Inc.) or $\beta$-actin antibody (Santa Cruz Biotechnology, Inc., Santa Cruz, CA, USA). The membranes were washed and further incubated for $1 \mathrm{~h}$ at $20^{\circ} \mathrm{C}$ with horseradish peroxidase-conjugated secondary antibodies (ZSGB-BIO, Beijing, China). Following washing, the immunoreactive bands were visualized using a 3,3'-diami- nobenzidine kit (ZSGB-BIO). The results were analyzed by the UVP System (UVP, Upland, California, USA).

Statistical analysis. Results are expressed as the mean \pm standard deviation. The statistical significance of differences between the groups was determined by one-way analysis of variance using SPSS 13.0 statistical software (SPSS, Inc., Chicago, IL, USA). Fisher's exact test and $\chi^{2}$ test were used to select the significant pathways and $\mathrm{P}<0.05$ was considered to indicate a statistically significant difference.

\section{Results}

Cell aggregates are proliferation-resistant. The detached cells gathered to form aggregates (Fig. 1A). The majority of cells in aggregates were alive, with the death rate $<5 \%$ (Fig. 1B). The proliferation ability of cells in cell aggregates was markedly lower in the detached group than in the attached control group, as determined by a CCK8 assay (Fig. 1C).

MAPK pathway is upregulated to promote the survival of cells in cell aggregates. The MAPK signaling pathway was important in the different expression pathway networks (Fig. 2A). The indegree and outdegree of MAPK signaling pathway were 5 and 1 , respectively. It was one of the most important pathways in the entire pathway network. Five genes in this pathway, including FOS, DDIT3 and MST1, were upregulated when the cells were detached and gathered to form aggregates. In the conjoint analysis of Signal-Net and microRNA-Gene-Network, FOS, DDIT3 and MST1 in 


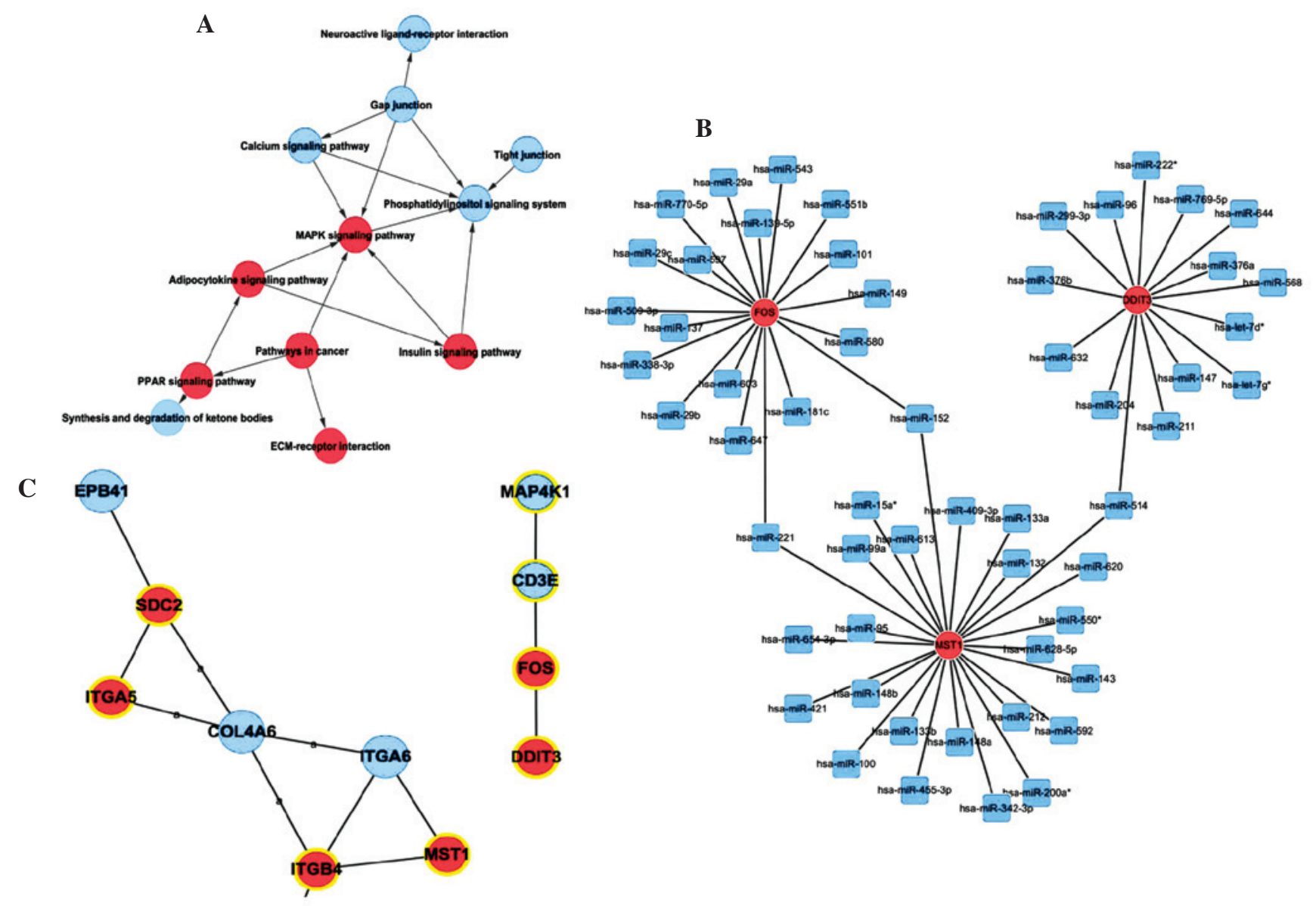

Figure 2. The mitogen-activated protein kinase (MAPK) signaling pathway was upregulated as determined by the microarray analysis. (A) The Signal-Net of the differentially expressed pathways in detached BEL7402 human hepatoma cells compared with the attached control group. Red circles represent upregulated pathways and blue circles represent downregulated pathways. The MAPK signaling pathway, with 5 indegree and 1 outdegree, was a key intermediate signaling pathway in the network. (B) Conjoint analysis of Signal-Net and microRNA-Gene-Network. Red circles represent upregulated genes and blue circles represent downregulated genes. Genes that existed in the microRNA-Gene-Network are marked with yellow circles. FOS, DDIT3 and MST1 in the MAPK signaling pathway existed in the two networks, which indicated that they were important in the anoikis-resistant progress. (C) The microRNA-Gene-Network of differentially expressed genes in the MAPK pathway. The blue squares represent downregulated microRNAs and the red circles represent upregulated genes. FOS, DDIT3 and MST1 were regulated by several microRNAs. For example, miR-221 regulated FOS and MST1, while miR-514 regulated MST1 and DDIT3.
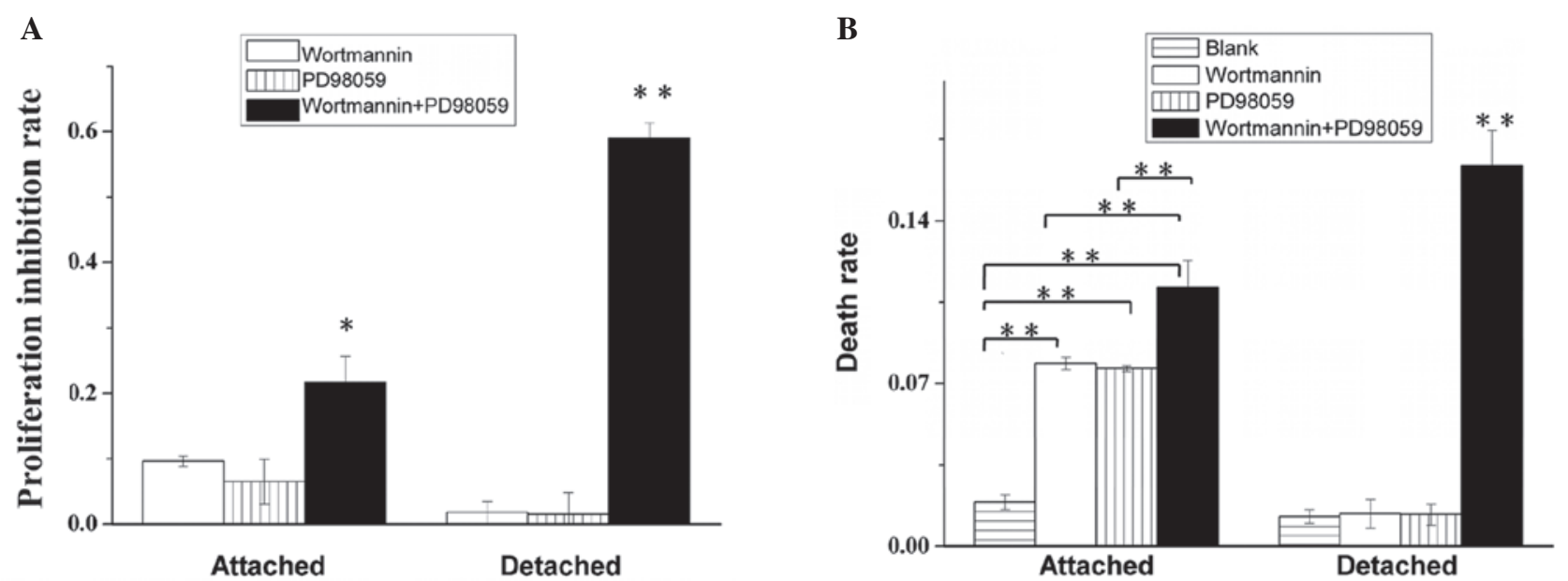

Figure 3. The response of detached BEL7402 human hepatoma cells to the inhibitors of AKT or extracellular signal-regulated kinase (ERK), wortmannin and PD98059, respectively. The viability of attached and detached BEL7402 cells treated in triplicate with either $1 \mu \mathrm{M}$ wortmannin, $50 \mu \mathrm{M}$ PD98059 or both for $24 \mathrm{~h}$ was detected by a cell counting kit-8 assay. (A) Using Wortmannin and PD98059 simultaneously induced markedly higher proliferation inhibition than using them separately. The increase of inhibitory rates when using the two inhibitors as opposed to using only one was greater in detached cells $\left({ }^{* *} \mathrm{P}<0.01\right)$ than in attached cells ( $\mathrm{P}<0.05)$. (B) The death rates of attached or detached BEL7402 hepatoma cells were detected by a trypan blue assay. Attached cells were markedly more sensitive than detached cells to Wortmannin or PD98059 when used separately. However, when these two inhibitors were used simultaneously, markedly more dead cells were observed in the detached group than in the attached group. Data are presented as the mean \pm standard deviation of one representative experiment from three independent experiments. ${ }^{*} \mathrm{P}<0.05,{ }^{* *} \mathrm{P}<0.01$. 
A
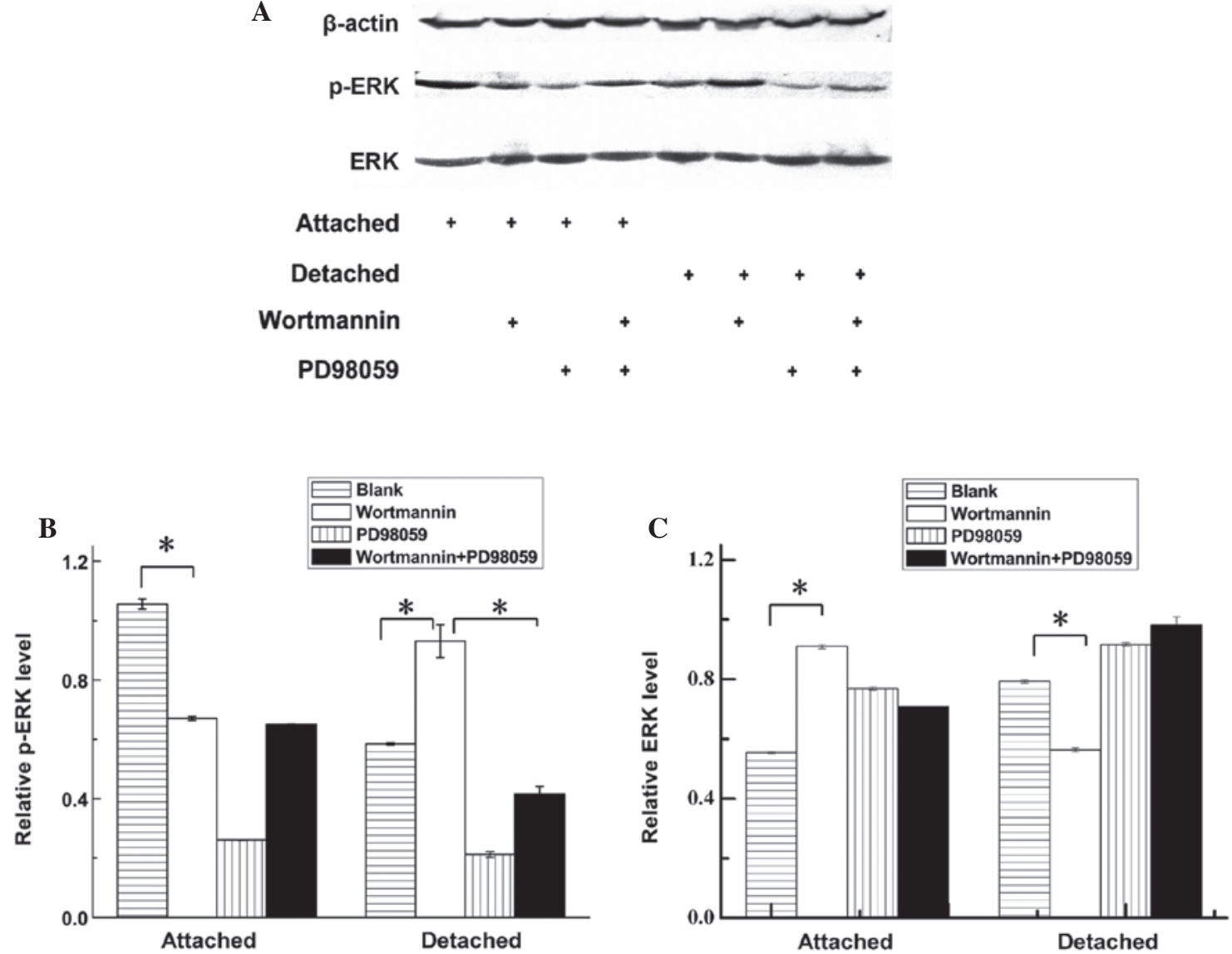

Figure 4. Compensatory activation of extracellular signal-regulated kinase (ERK) in anoikis-resistant hepatoma cells upon inhibition of the phosphatidylinositol 3-kinase (PI-3K)/AKT pathway with wortmannin and PD98059. BEL7402 human hepatoma cells in either adherent or suspension conditions were treated in triplicate with $1 \mu \mathrm{M}$ wortmannin, $50 \mu \mathrm{M}$ PD98059 or both for $24 \mathrm{~h}$. The ERK and phospho (p)-ERK protein levels were then detected by western blot analysis. (A) The ERK and p-ERK western blotting results for the different groups. The images shown are representative of three experiments performed for each experimental condition. (B) The relative p-ERK protein level of attached and detached hepatoma cells. The p-ERK protein level was upregulated in detached cells when Wortmannin was used to inhibit the AKT pathway. (C) The relative ERK protein level of attached and detached hepatoma cells. The ERK protein level was downregulated in detached cells and upregulated in attached cells when Wortmannin was used to inhibit the AKT pathway. Data are presented as the mean \pm standard deviation of three independent experiments. ${ }^{*} \mathrm{P}<0.05$.

the MAPK signaling pathway existed in the two networks (Fig. 2B). The microRNA-Gene-Network of differentially expressed genes in the MAPK pathway, revealed that FOS, DDIT3 and MST1 were regulated by several microRNAs (Fig. 2C). For example, miR-221 regulated FOS and MST1, while miR-514 regulated MST1 and DDIT3.

Anoikis-resistant suspended cells are less sensitive to AKT and ERK inhibitors. The growth of attached BEL7402 cells was greatly inhibited by the effect of AKT or ERK inhibition (Fig. 3A). However, the detached BEL7402 cells were markedly more resistant to AKT or ERK inhibition. Notably, when the AKT and ERK pathways were inhibited together, the viability of the detached cells decreased markedly, much more than in the attached groups. The death rates of attached and detached BEL7402 hepatoma cells were detected by a trypan blue assay (Fig. 3B). The cell death rate of detached cells treated with Wortmannin or PD98059 separately was markedly lower than in the attached control. However, when the inhibitors were used together, the death rate of detached cells increased markedly, to a significantly greater degree than that in the attached control group.
Compensatory activation of ERK in anoikis-resistant hepatoma cells upon inhibition of the PI-3K/AKT pathway. The ERK compensatory activation of the PI-3K/AKT pathway inhibited the detached cell group and was confirmed by western blot analysis. The ERK and phospho (p)-ERK western blotting results in different groups are shown in Fig. 4A. The relative p-ERK protein level of attached and detached hepatoma cells indicates that the p-ERK protein level was upregulated in detached cells when Wortmannin was used to inhibit the AKT pathway (Fig. 4B). The relative ERK protein level of attached and detached hepatoma cells indicates that ERK protein was downregulated in detached cells but upregulated in attached cells when Wortmannin was used to inhibit the AKT pathway (Fig. 4C).

\section{Discussion}

Metastasis is an important factor in malignancy and is commonly responsible for the failure of cancer treatment. How cancer cells manage to survive during the entire metastatic process and succeed in localizing to a secondary location remains controversial. Our previous study revealed 
that hepatoma cells prevented anoikis through synoikis-like survival (8). Acquisition of anoikis resistance caused the detached hepatoma cells to develop further malignant properties and acquire the metastatic potential to form metastatic cancer at the secondary microenvironment (15).

In the present study, microarray was used to elucidate the molecular mechanism that may be involved in the proliferation inhibition of detached hepatoma cells. The results indicated that the MAPK signaling pathway is upregulated in anoikis-resistant cells. MAPK signaling pathway upregulation usually leads to cell proliferation activity, which is contrary to the proliferation inhibition of detached hepatoma cells, a process validated in the current and in previous studies (8). This contradiction is noteworthy and further investigation is required.

To determine whether a downstream PI-3K/AKT or MAPK pathway is required for the survival of metastatic hepatoma cells in suspension, the response of attached and detached BEL7402 cells to PI-3K/AKT and MAPK pathway inhibitors was evaluated. The results revealed that when the PI-3K/AKT and MAPK pathways were inhibited separately, the effect on the viability of the anoikis-resistant cells was weak. By contrast, when these two protein kinase pathways were inhibited simultaneously, extensive cell death ensued. These data indicate that there is a clear interplay between these two prosurvival pathways. It is possible that when one of these two pathways was inhibited, the other pathway was overactivated and compensated for the loss of this survival signal. Notably, the western blot analysis revealed that inhibition of the PI-3K/AKT pathway markedly increased the phosphorylation level of the ERK protein. This result validates the hypothesis that crosstalk exists between these two signaling pathways. It was previously reported that inhibition of AKT activation resulted in a marked increase in the ERK pathway phosphorylation when apoptosis was induced by DNA-damaging drugs (33). However, no report has yet confirmed this compensatory activation in metastatic hepatoma cells when one of the survival pathways is inhibited. The marked activation of ERK pathway phosphorylation may offer further survival opportunities for hepatoma cells following deprivation of anchorage, which may also contribute to their metastatic potential. Whether the anoikis resistance is dependent on AKT or ERK activation remains controversial and the function of these prosurvival pathways in resisting detachment-induced cell death remains a matter of debate (34-37).

The MAPK signaling pathway is considered to be fundamental in the regulation of proliferation in mammalian cells by sharing a substrate and cross-cascade interaction with other signal transduction systems. Understanding this pathway is therefore essential for the rational design of novel pharmacotherapeutic approaches (38). Notably, modulation of the PI-3K/AKT and MAPK signaling pathways has also been reported to influence apoptotic responses to anticancer drugs. The data from the present study indicate that due to the powerful interplay among cell signaling pathways, using a combination of kinase inhibitors may yield a substantial advance in successfully producing a downstream phenotypic response in anoikis-resistant hepatoma cells. This is in accordance with the hypothesis that a combination of drugs that target different aspects of the metastatic process may be a therapeutic strategy in the future (2). However, the mechanism of this powerful interplay remains unknown. The functions of FOS, DDIT3, MST1, miR-221 and miR-514 may be investigated to analyze the underlying metastatic mechanisms in future studies.

\section{Acknowledgements}

This study was supported by grants from the Natural Science Foundation of China (grant nos. 30700357, 30772031 and 30873025) and the Natural Science Foundation of Shandong Province (grant no. ZR2010HM033).

\section{References}

1. Llovet JM, Burroughs A, Bruix J: Hepatocellular carcinoma. Lancet 362: 1907-1917, 2003.

2. Mazzocca A and Carloni V: The metastatic process: methodological advances and pharmacological challenges. Curr Med Chem 16: 1704-1717, 2009.

3. Sleeman J and Steeg PS: Cancer metastasis as a therapeutic target. Eur J Cancer 46: 1177-1180, 2010.

4. Loberg RD, Fridman Y, Pienta BA, Keller ET, McCauley LK, Taichman RS and Pienta KJ: Detection and isolation of circulating tumor cells in urologic cancers: a review. Neoplasia 6: 302-309, 2004

5. Simpson CD, Anyiwe K and Schimmer AD: Anoikis resistance and tumor metastasis. Cancer Lett 272: 177-185, 2008.

6. Frisch SM: Evidence for a function of death-receptor-related, death-domain-containing proteins in anoikis. Curr Biol 9: 1047-1049, 1999

7. Derouet M, Wu X, May L, Hoon Yoo B, Sasazuki T, Shirasawa S, et al: Acquisition of anoikis resistance promotes the emergence of oncogenic K-ras mutations in colorectal cancer cells and stimulates their tumorigenicity in vivo. Neoplasia 9: 536-545, 2007.

8. Zhang Z, Cao L, Li J, Liang X, Liu Y, Liu H, et al: Acquisition of anoikis resistance reveals a synoikis-like survival style in BEL7402 hepatoma cells. Cancer Lett 267: 106-115, 2008.

9. Geiger TR and Peeper DS: Critical role for TrkB kinase function in anoikis suppression, tumorigenesis, and metastasis. Cancer Res 67: 6221-6229, 2007.

10. Zhang Y, Lu H, Dazin P and Kapila Y: Squamous cell carcinoma cell aggregates escape suspension-induced, p53-mediated anoikis: fibronectin and integrin alphav mediate survival signals through focal adhesion kinase. J Biol Chem 279: 48342-48349, 2004.

11. Frisch SM and Francis H: Disruption of epithelial cell-matrix interactions induces apoptosis. J Cell Biol 124: 619-626, 1994.

12. Frisch SM and Screaton RA: Anoikis mechanisms. Curr Opin Cell Biol 13: 555-562, 2001.

13. Christofori G: Changing neighbours, changing behaviour: cell adhesion molecule-mediated signalling during tumour progression. EMBO J 22: 2318-2323, 2003.

14. Hanahan D and Weinberg RA: The hallmarks of cancer. Cell 100: 57-70, 2000.

15. Cao L, Han L, Zhang Z, Li J, Qu Z, Du J, et al: Involvement of anoikis-resistance in the metastasis of hepatoma cells. Exp Cell Res 315: 1148-1156, 2009.

16. Aplin AE, Howe A, Alahari SK and Juliano RL: Signal transduction and signal modulation by cell adhesion receptors: the role of integrins, cadherins, immunoglobulin-cell adhesion molecules, and selectins. Pharmacol Rev 50: 197-263, 1998.

17. Giancotti FG and Ruoslahti E: Integrin signaling. Science 285 : 1028-1032, 1999.

18. Douma S, Van Laar T, Zevenhoven J, Meuwissen R, Van Garderen E and Peeper DS: Suppression of anoikis and induction of metastasis by the neurotrophic receptor TrkB. Nature 430: 1034-1039, 2004.

19. Zhan M, Zhao H and Han ZC: Signalling mechanisms of anoikis. Histol Histopathol 19: 973-983, 2004.

20. Saxena NK, Sharma D, Ding X, Lin S, Marra F, Merlin D and Anania FA: Concomitant activation of the JAK/STAT, $\mathrm{PI} 3 \mathrm{~K} / \mathrm{AKT}$, and ERK signaling is involved in leptin-mediated promotion of invasion and migration of hepatocellular carcinoma cells. Cancer Res 67: 2497-2507, 2007. 
21. Díaz-Montero CM, Wygant JN and McIntyre BW: $\mathrm{PI} 3-\mathrm{K} / \mathrm{Akt}$-mediated anoikis resistance of human osteosarcoma cells requires Src activation. Eur J Cancer 42: 1491-1500, 2006.

22. Horowitz JC, Rogers DS, Sharma V, Vittal R, White ES, Cui Z and Thannickal VJ: Combinatorial activation of FAK and AKT by transforming growth factor-betal confers an anoikis-resistant phenotype to myofibroblasts. Cell Signal 19: 761-771, 2007.

23. Collins NL, Reginato MJ, Paulus JK, Sgroi DC, Labaer J and Brugge JS: G1/S cell cycle arrest provides anoikis resistance through Erk-mediated Bim suppression. Mol Cell Biol 25 $5282-5291,2005$

24. Fukazawa H, Noguchi K, Murakami Y and Uehara Y: Mitogen-activated protein/extracellular signal-regulated kinase kinase (MEK) inhibitors restore anoikis sensitivity in human breast cancer cell lines with a constitutively activated extracellular-regulated kinase (ERK) pathway. Mol Cancer Ther 1: 303-309, 2002

25. Kanehisa M, Goto S, Kawashima S, Okuno Y and Hattori M: The KEGG resource for deciphering the genome. Nucleic Acids Res 32: D277-D280, 2004

26. Yi M, Horton JD, Cohen JC, Hobbs HH and Stephens RM: WholePathwayScope: a comprehensive pathway-based analysis tool for high-throughput data. BMC Bioinformatics 7: 30, 2006.

27. Draghici S, Khatri P, Tarca AL, Amin K, Done A, Voichita C, et al A systems biology approach for pathway level analysis. Genome Res 17: 1537-1545, 2007.

28. Jansen R, Greenbaum D and Gerstein M: Relating whole-genome expression data with protein-protein interactions. Genome Res 12: 37-46, 2002.

29. $\mathrm{Li} \mathrm{C}$ and $\mathrm{Li} \mathrm{H}$ : Network-constrained regularization and variable selection for analysis of genomic data. Bioinformatics 24 $1175-1182,2008$
30. Wei $\mathrm{Z}$ and Li H: A Markov random field model for network-based analysis of genomic data. Bioinformatics 23: 1537-1544, 2007.

31. Zhang JD and Wiemann S: KEGGgraph: a graph approach to KEGG PATHWAY in R and bioconductor. Bioinformatics 25: 1470-1471, 2009.

32. Spirin V and Mirny LA: Protein complexes and functional modules in molecular networks. Proc Natl Acad Sci USA 100: 12123-12128, 2003.

33. Lee ER, Kim JY, Kang YJ, Ahn JY, Kim JH, Kim BW, et al: Interplay between PI3K/Akt and MAPK signaling pathways in DNA-damaging drug-induced apoptosis. Biochim Biophys Acta 1763: 958-968, 2006 .

34. Rul W, Zugasti O, Roux P, Peyssonnaux C, Eychene A, Franke TF, et al: Activation of ERK, controlled by Racl and Cdc42 via Akt, is required for anoikis. Ann N Y Acad Sci 973 145-148, 2002.

35. Ishida K, Nagahara H, Kogiso T, Aso T, Hayashi $\mathrm{N}$ and Akaike T: Cell adhesion aside from integrin system can abrogate anoikis in rat liver cells by down-regulation of FasL expression, not by activation of PI-3K/Akt and ERK signaling pathway. Biochem Biophys Res Commun 300: 201-208, 2003.

36. Choi EM, Kwak SJ, Kim YM, Ha KS, Kim JI, Lee SW and Han SJ: COX-2 inhibits anoikis by activation of the PI-3K/Akt pathway in human bladder cancer cells. Exp Mol Med 37: 199-203, 2005.

37. Zugasti O, Rul W, Roux P, Peyssonnaux C, Eychene A, Franke TF, et al: Raf-MEK-Erk cascade in anoikis is controlled by Rac1 and Cdc42 via Akt. Mol Cell Biol 21: 6706-6717, 2001.

38. Zhang W and Liu HT: MAPK signal pathways in the regulation of cell proliferation in mammalian cells. Cell Res 12: 9-18, 2002. 